\section{Radiopharmaceuticals for Therapy}

\author{
F.F. Knapp and A. Dash
}

New York, NY: Springer, 2016, 347 pages, $\$ 109$

There is no denying that the next phase of growth for nuclear medicine is in radionuclide therapy. Radiopharmaceuticals for Therapy is a well-executed book, divided into 5 parts with the primary focus on the use of radioisotopes for the treatment of disease. The first 2 chapters provide an overview of radiotherapy and are geared toward a broad science-based audience. In chapter 1, the authors lay the foundation and set the stage for the book by introducing the field of nuclear medicine and discussing how radiopharmaceuticals are used in both diagnostic and therapeutic applications, with Table 1.1 highlighting the key radionuclides of nuclear medicine. The authors then shift into a discussion of therapeutic radiopharmaceuticals, recognizing the renewed interest in the development of such agents that has been brought about by the recent Food and Drug Administration approval of ${ }^{223}$ Ra-dichloride (Xofigo; Bayer) for castration-resistant prostate cancer. Chapter 2 deliberates the criteria for the selection of therapeutic radionuclides, noting that successful production and procurement of radionuclides is the first step in the assessment of their utility for therapeutic applications. The authors explain that the underlying principle of radiotherapy is either to ablate or to damage diseased tissue through the emissions of $\beta$-particles, $\alpha$-particles, or Auger electrons.

Chapter 3 briefly gives a historical background on the development of $\alpha$-radionuclide therapy and then transitions into a discussion of the rapidly increasing interest in $\alpha$-emitters stemming from the approval of Xofigo. Table 3.1 outlines the key $\alpha$-emitting radionuclides for therapy, and in the chapter summary, the authors recognize the barriers to the widespread use of these agents. Chapter 4 explores the Auger emitters, which are an alternative to the use of $\alpha$-emitting and $\beta$-emitting radioisotopes for therapy. Table 4.1 provides a helpful comparison of energy emission and softtissue penetration between $\alpha$ - and $\beta$-particles and the Auger electrons.

Part II is an up-to-date review and evaluation of the production of therapeutic radionuclides. This part of the book is geared more toward the radiochemist. Its 4 chapters cover reactor-produced agents such as ${ }^{177} \mathrm{Lu}$, accelerator-produced agents such as ${ }^{225} \mathrm{Ac}$ and ${ }^{223} \mathrm{Ra}$, generator-produced agents such as ${ }^{90} \mathrm{Sr} /{ }^{90} \mathrm{Y}$, and agents produced via decay of naturally occurring parents, such as production of ${ }^{223} \mathrm{Ra}$ from ${ }^{227} \mathrm{Ac}$ and ${ }^{227} \mathrm{Th}$. The detailed discussion of the production of each radioisotope is crucial to help readers understand the true limitations to the widespread use of some radiopharmaceuticals for therapy.

The remainder of the book focuses on the therapeutic applications of particular radiopharmaceuticals. The first 2 chapters of part III deal with agents used to treat cancer, such as radiolabeled antibodies (radioimmunotherapy) and peptide receptor radionuclide therapy. Table 9.7 gives examples of radionuclides for radioimmunotherapy, and Table 10.4 lists essential radionuclides for peptide receptor radionuclide therapy. The last chapter of part III touches on therapeutic radiopharmaceuticals for hepatocellular carcinoma, which is the sixth most common cancer worldwide. Again, the authors provide a helpful table (Table 11.3), this one depicting the relevance of the various radionuclides used to treat hepatocellular carcinoma.

Part IV describes and assesses therapeutic radiopharmaceuticals for chronic diseases, including agents for palliation of bone pain, agents for treatment of nonmelanoma skin cancer and synovial joint inflammation, and intravascular radiation therapy for arterial restenosis.

In part $\mathrm{V}$, the authors conclude the book with a dialog on opportunities for new therapeutic agents, namely nanoparticlebased agents targeted for delivery to diseased cells, stating that these agents will take us closer to the realm of personalized medicine. The final chapter provides a synopsis of the "complex maze of regulatory and marketing requirements" that must be traversed to move a drug from bench to bedside-from development on the laboratory bench to introduction to the clinical setting. The information presented in the final chapter is strategic in helping readers understand the limitations of therapeutic radiopharmaceutical development and, thus, application.

Radiopharmaceuticals for Therapy is a comprehensive volume on radiotherapeutics and unquestionably delivers on its promise to be highly useful to the nuclear medicine professional.

Published online May 25, 2017.
DOI: 10.2967/jnumed.117.196568

\author{
Dao Le \\ M.D. Anderson Cancer Center \\ 1515 Holcombe Blvd. \\ Houston, TX 77030 \\ E-mail:dble@mdanderson.org.
}

